01

\title{
Сложная динамика неавтономного осциллятора с управляемой фазой внешнего воздействия
}

\author{
(C) Е.П. Селезнев ${ }^{1,2}$, Н.В. Станкевич $1,3,4$, ฯ \\ ${ }^{1}$ Саратовский филиал Института радиотехники и электроники им. В.А. Котельникова РАН, Саратов, Россия \\ ${ }^{2}$ Саратовский государственный университет им. Н.Г. Чернышевского, Саратов, Россия \\ ${ }^{3}$ Саратовский государственный технический университет им. Гагарина Ю.А., Саратов, Россия \\ ${ }^{4}$ Санкт-Петербургский государственный университет, Санкт-Петербург, Россия \\ I E-mail: stankevichnv@mail.ru
}

Поступило в Редакцию 25 июля 2018 г.

Исследуется динамика неавтономного осциллятора, в котором фаза внешнего воздействия зависит линейно от динамической переменной. Такое управление фазой внешнего воздействия приводит к тому, что в поведении осциллятора наблюдается иерархия различных периодических и хаотических колебаний. Изучена структура пространства управляющих параметров. Показано, что в динамике системы наблюдаются колебательные режимы, аналогичные режимам неавтономного осциллятора с потенциалом в виде периодической функции.

DOI: 10.21883/PJTF.2019.02.47227.17473

Во многих системах, включая радиофизические, биологические и др., наблюдаются колебательные процессы, при которых один объект, входящий в систему, воздействует на другой периодическим сигналом, но при изменении условий функционирования частота воздействия изменяется. Например, в системах передачи информации для обеспечения высокой устойчивости используется так называемая фазовая автоподстройка частоты [1]. Система сердечно-сосудистой регуляции живых организмов при изменении нагрузки увеличивает или уменьшает частоту сердцебиений [2]. При такого рода взаимодействиях зависимость фазы от динамической переменной может привести к возникновению в системе сложной динамики.

В качестве простейшего объекта исследования такой системы выберем классическую модель теории колебаний [3] $-R L C$-контур, возбуждаемый внешним колебанием:

$$
\ddot{x}+2 \alpha \dot{x}+\omega_{0}^{2} x=V \sin (\omega t+\varphi)
$$

где $x, \dot{x}-$ динамические переменные, $\alpha-$ коэффициент диссипации, $\omega_{0}$ - собственная частота колебаний контура, $V, \omega$ и $\varphi-$ амплитуда, частота и фаза внешнего воздействия. Если $\varphi=$ const, то уравнение (1) описывает поведение линейного неавтономного осциллятора. Если фаза зависит от динамической переменной, то уравнение (1) становится нелинейным. Рассмотрим наиболее простой вариант, когда фаза меняется по линейному закону

$$
\varphi(t)=k x(t)
$$

где $k$ - постоянный коэффициент, $x(t)$ - динамическая переменная уравнения (1).
После перехода к безразмерному времени $\tau=\omega_{0} t$ уравнение (1) принимает вид

$$
\ddot{x}+2 \alpha \dot{x}+x=V \sin (p \tau+k x),
$$

где $p=\omega / \omega_{0}-$ нормированная частота внешнего воздействия. По сравнению с предыдущими случаями уравнение (3) содержит нелинейность типа $\sin (k x)$. В такой системе возможно появление сложных периодических и хаотических колебаний [4-7]. Особо отметим недавнюю работу [8], в которой было показано формирование мультистабильных хаотических аттракторов в неавтономной системе с синусоидальной нелинейностью.

Перейдем к численному исследованию системы (3). Анализ характера вынужденных колебаний осуществлялся на основе оценки спектра показателей Ляпунова, а также с помощью анализа сечения Пуанкаре. В качестве управляющих параметров использовались амплитуда $V$, частота $p$ и коэффициент фазовой перестройки $k$. Для анализа на устойчивость уравнение (3) было преобразовано в систему трех дифференциальных уравнений первого порядка

$$
\begin{gathered}
\dot{x}=y, \\
\dot{y}=-2 \alpha y-x+V \sin z, \\
\dot{z}=p+k y .
\end{gathered}
$$

На рис. 1, $а$ представлена карта динамических режимов системы (4) на плоскости параметров $(k, p)$ при значении амплитуды воздействия $V=1$. Различными оттенками серого цвета обозначены области периодических колебаний в соответствии с палитрой, представленной на рис. 1 внизу; хаотические колебания отмечены черным цветом. Штриховыми линиями отмечены резонансная и кратные ей частоты системы (3). Как видно из рис. 1, $a$, область сложных колебаний ограничена частотами воздействия, значения которых 


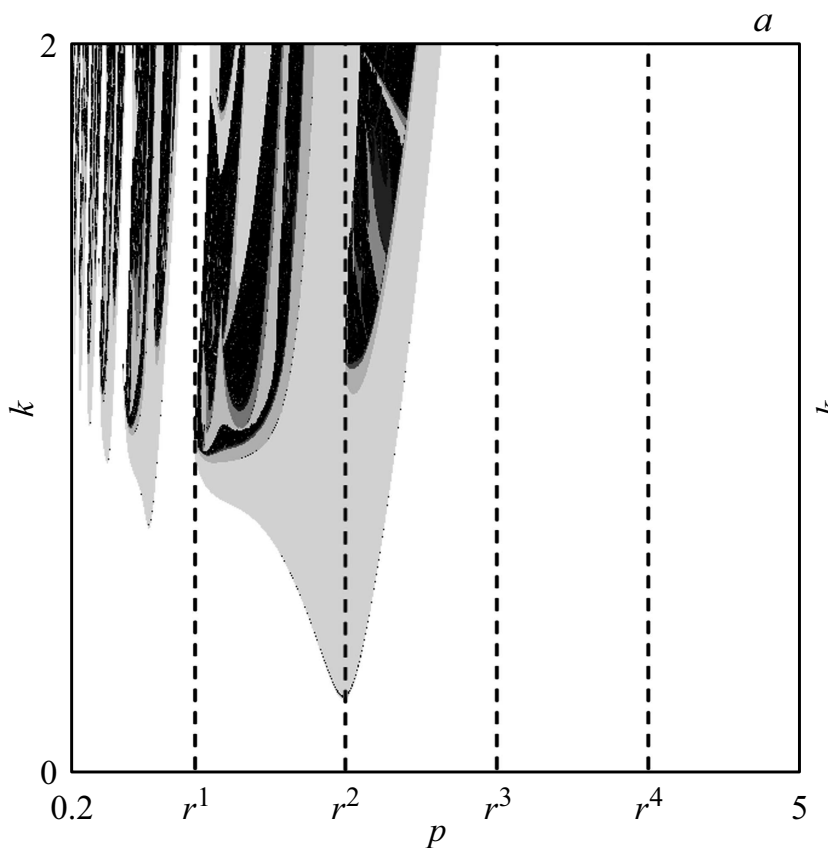

Periodic oscillations

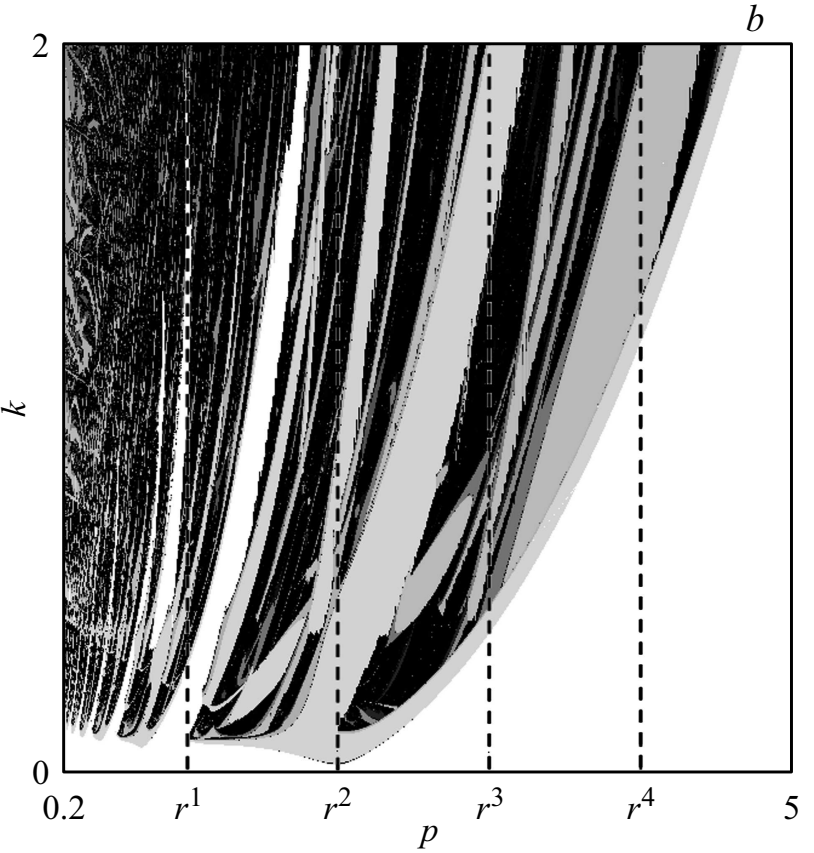

Chaotic oscillations

\section{\begin{tabular}{|l|l|l|l|l|l|l|l|l|l|l|l|l|}
\hline 1 & 2 & 3 & 4 & 5 & 6 & 7 & 8 & 9 & 10 & 11 & 12 & \\
\hline
\end{tabular}}

Рис. 1. Карты динамических режимов модели (4) при $\alpha=0.1 . V=1(a)$ и $10(b)$.

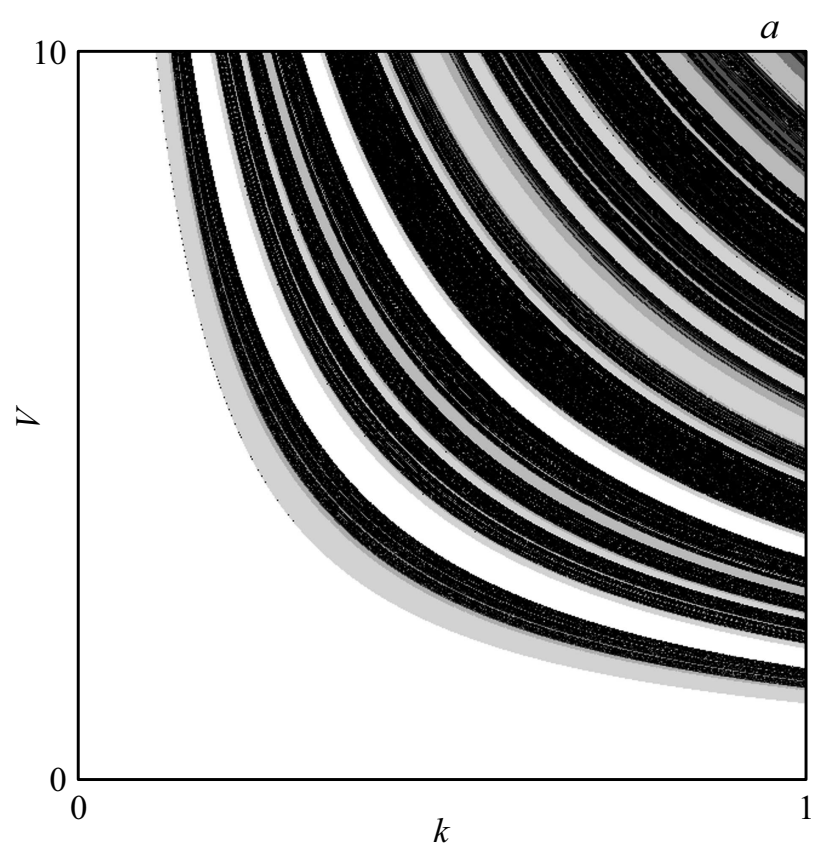

Periodic oscillations

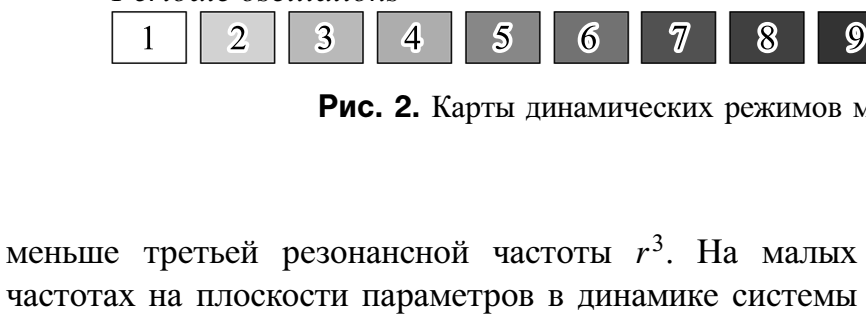
наблюдается последовательность бифуркаций удвоения периода, завершающаяся переходом к хаосу. Линии

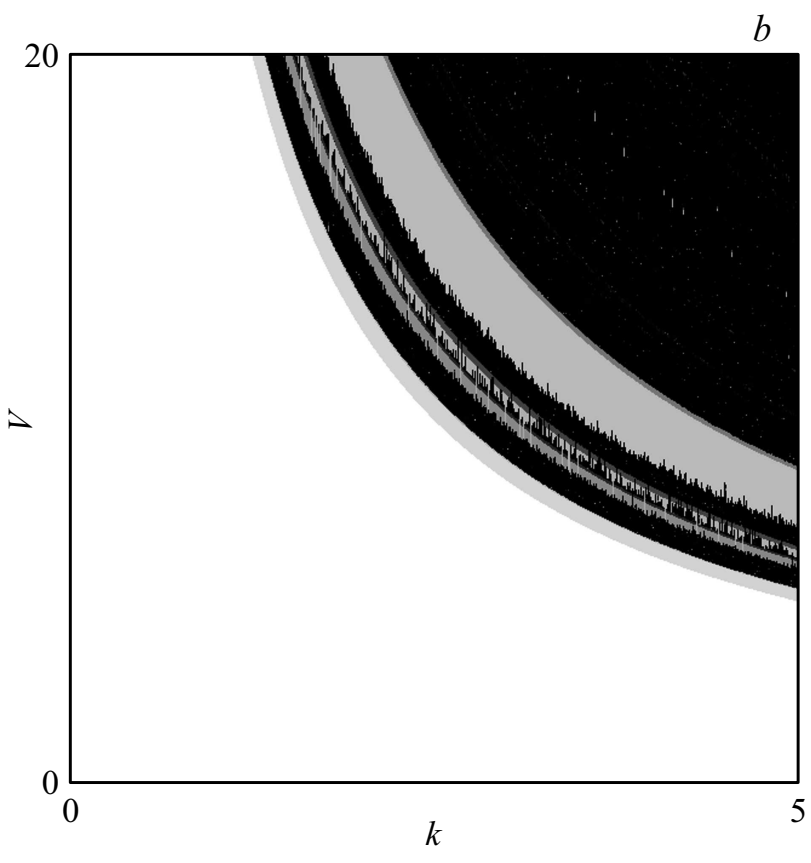

Chaotic oscillations

\section{2}

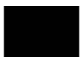



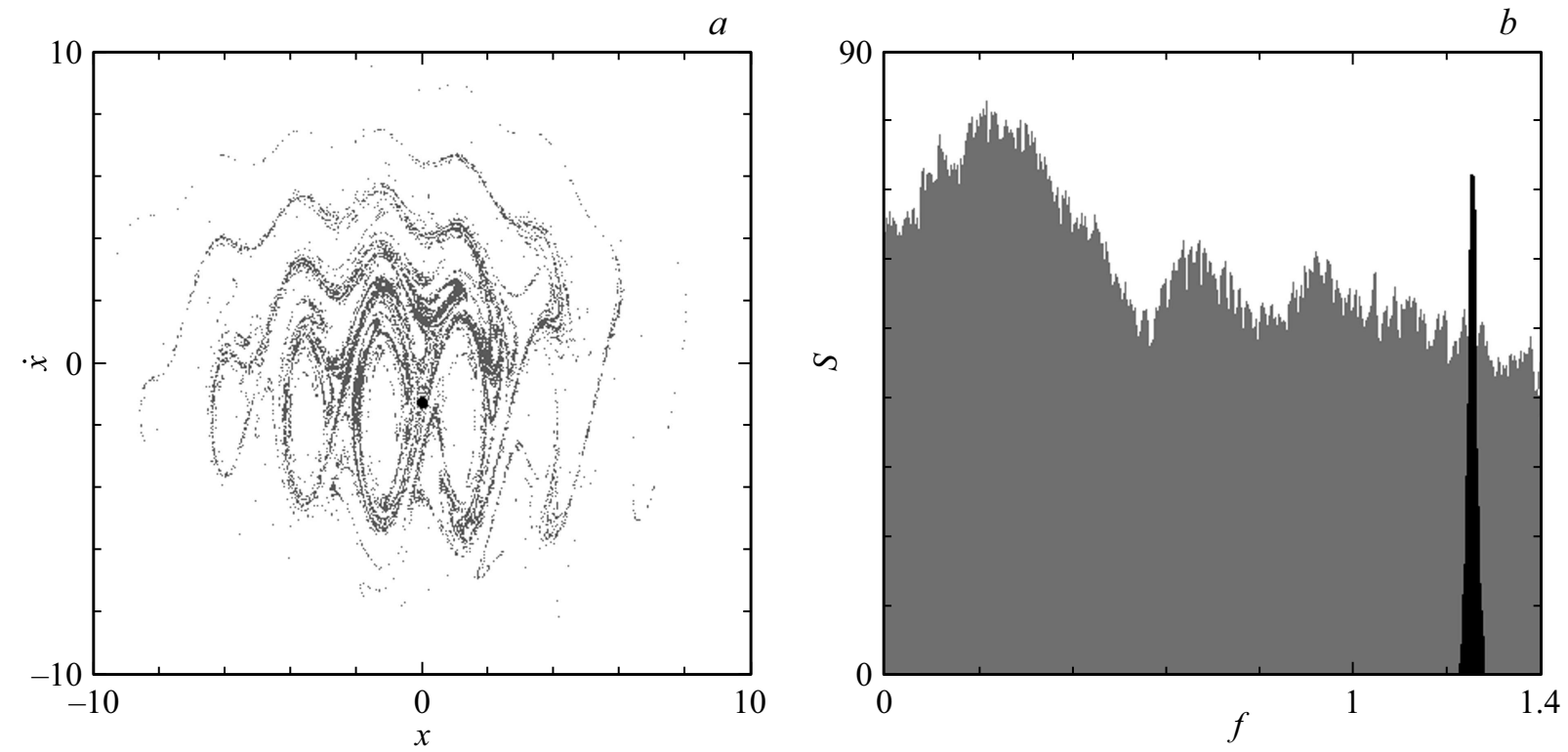

Рис. 3. $a-$ фазовый портрет модели (4) в стробоскопическом сечении при $\alpha=0.1, p=5, k=2.5, V=8.4, x_{0}=-0.0267$, $y_{0}=-1.2176$ (черный цвет - периодический режим) и $x_{0}=2.1878, y_{0}=-4.8897$ (серый цвет - хаотический режим); $b-$ спектры Фурье сосуществующих режимов.

для структуры пространства управляющих параметров неавтономного нелинейного осциллятора [2-7]. С уменьшением частоты воздействия наблюдаются аналогичные линии удвоения периода на частотах, отвечающих субрезонансам. С уменьшением частоты порог бифуркации удвоения периода увеличивается.

С увеличением параметра $k$ наблюдается развитие хаоса, обусловленное уменьшением связности хаотического аттрактора, чередуемое с появлением зон периодических колебаний. В целом структура плоскости управляющих параметров (рис. 1,a) во многом напоминает аналогичную для неавтономного нелинейного осциллятора [6,7]: можно выделить отдельные зоны сложного поведения, связанные с так называемыми резонансами на высших гармониках.

На рис. $1, b$ представлена карта режимов колебаний системы (4) на плоскости параметров $(k, p)$ при значении амплитуды воздействия $V=10$. Из сравнения с рис. $1, a$ видно, что увеличение амплитуды привело к расширению областей существования сложных колебательных режимов, а также к появлению в области существования хаоса новых зон периодических колебаний. Но минимум первой линии бифуркации удвоения периода по-прежнему находится на удвоенной резонансной частоте $r^{2}$, как и при малой амплитуде. Возникновение зон периодических режимов в области существования хаоса носит жесткий характер. При этом в динамике системы появляются режимы колебаний, соответствующие так называемой динамике нелинейного осциллятора c „многоямной““ потенциальной функцией. В частности, подобная динамика наблюдается в обобщенном уравнении Дуффинга [2-5]. Переход от периодического режима к хаосу также происходит через последовательность бифуркаций удвоения периода.
Обратимся к анализу плоскости параметров амплитуда воздействия-фазовая расстройка. На рис. 2, $a$ и $b$ представлены карты динамических режимов на плоскости параметров $(V, k)$ для двух значений параметра $p: p=0.25(a)$ и $5(b)$. С ростом $V$ и $k$ в поведении исследуемой системы (рис. 2,a) наблюдается переход к хаосу через последовательность удвоений периода колебаний. В области хаоса жестким образом формируются новые области периодических колебаний, переход от которых к хаосу также происходит через последовательность бифуркаций удвоения периода. При значении параметра $p=5$ (рис. $2, b$ ) структура карты параметров качественно повторяется, что иллюстрирует многообразие зон существования различных режимов колебаний. Как и на рис. $2, a$, с ростом параметров $V$ и $k$ в поведении исследуемой системы наблюдается переход к хаосу через последовательность удвоений периода колебаний; в области хаоса формируются новые области колебаний периодов 3 и 5; имеется обширная область хаоса с узкими зонами периодических колебаний.

При больших значениях параметра $p$ на карте хорошо видны жесткие переходы между различными динамическими режимами. Такая картина указывает на наличие в системе мультистабильности. Анализ системы на мультистабильность показал, что в системе возможно возникновение различных типов сосуществующих аттракторов. Могут сосуществовать периодические аттракторы с различным периодом, возможна также мультистабильность между периодическими и хаотическими колебаниями.

На рис. 3, а представлены сосуществующие аттракторы: с предельным циклом периода 1 (черный цвет) и хаотический аттрактор (серый цвет). На рисунке показаны аттракторы в стробоскопическом сечении, т. е. через период внешнего воздействия, построенные для 
одних и тех же параметров $\alpha=0.1, p=5, k=2.5$, $V=8.4$, но для различных начальных значений численного интегрирования: при $x_{0}=-0.0267, y_{0}=-1.2176$ фазовая траектория приходит на устойчивый предельный цикл периода 1 , а при $x_{0}=2.1878, y_{0}=-4.8897-$ на хаотический аттрактор. Как видно из рисунка, в стробоскопическом сечении предельному циклу соответствует одна неподвижная точка. Хаотический аттрактор представляет собой сложную структуру, аналогичную структуре аттрактора неавтономной системы в работе [8]. На рис. 3, $b$ представлены спектры Фурье сосуществующих режимов: по-прежнему серый цвет соответствует хаотическому режиму, черный - периодическому. Из рис. $3, b$ хорошо видно, что спектральный состав сигналов сильно различается. Основной пик хаотического колебания находится на более низких частотах, при этом спектр довольно широкий. Хаотический аттрактор характеризуется следующими показателями Ляпунова: $\Lambda_{1}=0.2332, \Lambda_{2}=0.0, \Lambda_{3}=-0.3431$.

Таким образом, введение линейной зависимости фазы внешнего воздействия от динамической переменной в неавтономном осцилляторе приводит к формированию в динамике системы иерархии периодических и хаотических колебаний. При малой амплитуде воздействия область существования сложных колебаний ограничена по частоте значением, примерно равным утроенной резонансной частоте. Увеличение амплитуды внешнего воздействия приводит к расширению областей существования сложных режимов колебаний, а также к появлению в области существования хаоса новых зон периодических колебаний. При этом в динамике системы появляются режимы колебаний, соответствующие так называемой динамике нелинейного осциллятора с периодическим потенциалом. Наблюдаются также мультистабильности.

Работа выполнена при финансовой поддержке РНФ (проект № 17-12-01008).

\section{Список литературы}

[1] Шалфеев В.Д., Матросов В.В. Нелинейная динамика систем фазовой синхронизации. Н. Новгород: Изд-во Нижегород. гос. ун-та, 2013. $336 \mathrm{c.}$

[2] Гайтон А.К., Холл Дж.Э. Медицинская физиология / Пер. с англ. под ред. В.И. Кобрина. М.: Логосфера, 2008. 1296 с.

[3] Рожнев А.Г., Трубецков Д.И. Линейные колебания и волны. М.: Физматлит, 2001. $416 \mathrm{c.}$

[4] Li T.Y., Yorke J.A. // Am. Math. Monthly. 1975. V. 82. P. 985992.

[5] Humieres D.D., Beasley M.R., Huberman B.A., Libhaber A. // Phys. Rev. A. 1982. V. 26. N 6. P. 3484-3496.

[6] Астахов В.В., Безручко Б.П., Селезнев Е.П. // Радиотехника и электроника. 1987. Т. 32. № 12. С. 2558-2566.

[7] Безручко Б.П., Селезнев Е.П. // Письма в ЖТФ. 1994. Т. 20. B. 19. C. $75-79$.

[8] Tang Y.-X., Khalaf A.J.M., Rajagopal K., Pham V.-T., Jafari S., Tian Y. // Chin. Phys. B. 2018. V. 27. N 4. P. 040502. 